CERN-TH/98-150

$\mathrm{DTP} / 97 / 104$

May 1998

\title{
Topology of Hadronic Flow for Higgs Production at Hadron Colliders
}

\author{
M. Heyssler ${ }^{a}$, V. A. Khoze ${ }^{a, b, c}$ and W. J. Stirling ${ }^{a, d}$ \\ ${ }^{a}$ Department of Physics, University of Durham, Durham DH1 3LE, England \\ ${ }^{b}$ INFN - Laboratori Nazionali di Frascati, I-00044 Frascati (Roma), Italy \\ ${ }^{c}$ TH Division, CERN, CH-1211 Geneva 23, Switzerland \\ ${ }^{d}$ Department of Mathematical Sciences, University of Durham, Durham DH1 3LE, \\ England
}

\begin{abstract}
Hadronic radiation provides a tool to distinguish different topologies of colour flow in hard scattering processes. We study the structure of hadronic flow corresponding to Higgs production and decay in high-energy hadron-hadron collisions. In particular, the signal $g g \rightarrow H \rightarrow b \bar{b}$ and background $g g \rightarrow b \bar{b}$ processes are shown to have very different radiation patterns, and this may provide an useful additional method for distinguishing Higgs signal events from the QCD background.
\end{abstract}




\section{Introduction}

The distribution of soft hadrons or jets accompanying energetic final-state particles in hard scattering processes is governed by the underlying colour dynamics at short distances [1 1 . The soft hadrons paint the colour portrait of the parton hard scattering, and can therefore act as a 'partonometer' [1 12]. Since signal and background processes at hadron colliders can have very different colour structures (compare for example the $s$-channel colour singlet process $q \bar{q} \rightarrow Z^{\prime} \rightarrow q^{\prime} \bar{q}^{\prime}$ with the colour octet process $\left.q \bar{q} \rightarrow g^{*} \rightarrow q^{\prime} \bar{q}^{\prime}\right)$, the distribution of accompanying soft hadronic radiation in the events can provide a useful additional diagnostic tool for identifying new physics processes.

Quite remarkably, because of the property of Local Parton Hadron Duality (see for example Refs. [2, 3, 13]) the distribution of soft hadrons can be well described by the amplitudes for producing a single additional soft gluon. This distribution takes the form of a soft 'antenna pattern' distribution multiplying the leading-order hard scattering matrix element squared. Confirmation of the validity of this approach comes from recent studies of the production of soft hadrons and jets accompanying large $E_{T}$ jet and $W+$ jet production by the CDF [14] and D0 collaborations [15] at the Fermilab Tevatron.

One of the most important physics goals of the CERN LHC $p p$ collider is the discovery of the Higgs boson [16]. Many scenarios, corresponding to different production and decay channels, have been investigated, see for example the studies reported in Refs. [17, 18]. While final states containing leptons and photons are relatively background free, they generally have very small branching ratios. In contrast, the more probable decay channels involving (heavy) quarks have large QCD backgrounds. The question naturally arises whether hadronic radiation patterns could help distinguish such signals from backgrounds. We have in mind the following type of scenario. Suppose an invariant mass peak is observed in a sample of (tagged) $b \bar{b}$ events. If these correspond to Higgs production, then the distribution of accompanying soft radiation in the event' will look very different from that expected in background QCD production of $b \bar{b}$ pairs. One could imagine, for example, comparing the topologies of the hadronic flows 'on and off resonance'.

In this study we will consider the hadronic radiation patterns for two of the standard Higgs processes at LHC: direct production $g g \rightarrow H \rightarrow b \bar{b}$ and associated production $q \bar{q}^{\prime} \rightarrow W H \rightarrow \ell \nu_{\ell} b \bar{b}$. Although the non-zero $b$-quark mass is largely irrelevant when computing the radiation patterns, we will also consider the case when the final-state quark mass is large, so that our analysis can also be applied for example to $H \rightarrow t \bar{t}$. Our Higgs analysis is a natural extension of the studies of Refs. [9, 19], where the antenna patterns for $Z^{\prime}$ in $p \bar{p}$ collisions and leptoquark production in $e p$ collisions were calculated and shown to be different from those of the QCD backgrounds.

The analysis presented here should be regarded as a 'first look' at the possibilities

\footnotetext{
${ }^{1}$ We take this to mean the angular distribution of hadrons or 'minijets' with energies of at most a few $\mathrm{GeV}$, well separated from the beam and final-state energetic jet directions.
} 
offered by hadronic flow patterns in searching for the Higgs. Of course, ultimately there is no substitute for a detailed Monte Carlo study including detector effects. However the results presented here indicate that the effects can be potentially large, and therefore that more detailed studies are definitely worthwhile.

The paper is organised as follows. In the following section we consider direct production and $q \bar{q}$ decay of the Higgs boson, first for massless and then for massive quarks. Section 3 extends the analysis to associated production and Section 4 presents our conclusions.

\section{Hadronic radiation patterns for signal and back- ground processes}

We begin by considering the hadronic radiation patterns for the signal $(g g \rightarrow H \rightarrow q \bar{q}+g)$ and background $(g g \rightarrow q \bar{q}+g)$ production of a massless $q \bar{q}$ pair. The impact of non-zero quark masses will be considered later. The radiation pattern is defined as the ratio of the $2 \rightarrow 3$ and $2 \rightarrow 2$ matrix elements using the soft-gluon approximation for the former. The dependence on the soft gluon momentum $k$ then enters via the eikonal factors ('antennae') 20

$$
[i j]=\frac{p_{i} p_{j}}{\left(p_{i} k\right)\left(p_{j} k\right)} .
$$

For the QCD background process $g\left(p_{1}\right) g\left(p_{2}\right) \rightarrow q\left(p_{3}\right) \bar{q}\left(p_{4}\right)+g(k)$ we have

$$
\begin{aligned}
\frac{1}{g_{s}^{6}}\left|\overline{\mathcal{M}}_{3}\right|^{2}(g g \rightarrow q \bar{q}+g) & =\frac{1}{2}\left(t^{2}+u^{2}\right)\left[\left(1-\frac{1}{N_{c}^{2}}\right) \frac{1}{t u}-\frac{2}{s^{2}}\right]\left\{\frac{N_{c}}{C_{F}}[12]+[34]\right\} \\
& -\frac{1}{8}\left(t^{2}+u^{2}\right)\left[\left(1-\frac{2}{N_{c}^{2}}\right) \frac{1}{t u}-\frac{2}{s^{2}}\right]\left\{\frac{N_{c}}{C_{F}}[12 ; 34]\right\} \\
& +\frac{1}{8}\left(t^{2}-u^{2}\right)\left[\frac{1}{t u}-\frac{2}{s^{2}}\right]\left\{\frac{N_{c}}{C_{F}}([14]+[23]-[13]-[24])\right\},
\end{aligned}
$$

with $s=\left(p_{1}+p_{2}\right)^{2}, t=\left(p_{1}-p_{3}\right)^{2}, u=\left(p_{1}-p_{4}\right)^{2}$, and

$$
[i j ; k l]=2[i j]+2[k l]-[i k]-[i l]-[j k]-[j l] .
$$

This is to be normalised by the matrix element for the leading-order scattering process $g\left(p_{1}\right) g\left(p_{2}\right) \rightarrow q\left(p_{3}\right) \bar{q}\left(p_{4}\right):$

$$
\frac{1}{g_{s}^{4}}\left|\overline{\mathcal{M}}_{2}\right|^{2}(g g \rightarrow q \bar{q})=\frac{1}{2}\left(t^{2}+u^{2}\right)\left[\frac{1}{N_{c}} \frac{1}{t u}-\frac{1}{C_{F}} \frac{1}{s^{2}}\right] .
$$


The antenna pattern is then

$$
\mathcal{R}^{Q C D}=g_{s}^{-2} \frac{\left|\overline{\mathcal{M}}_{3}\right|^{2}(g g \rightarrow q \bar{q}+g)}{\left|\overline{\mathcal{M}}_{2}\right|^{2}(g g \rightarrow q \bar{q})} .
$$

Note that because of the non-trivial colour structure of the leading-order Feynman diagrams, see Fig. 1(a), there is no simple factorisation of the eikonal factors. This is in contrast to the signal (Higgs) process, for which

$$
\mathcal{R}^{H}=g_{s}^{-2} \frac{\left|\overline{\mathcal{M}}_{3}\right|^{2}(g g \stackrel{H}{\rightarrow} q \bar{q}+g)}{\left|\overline{\mathcal{M}}_{2}\right|^{2}(g g \stackrel{H}{\rightarrow} q \bar{q})}=2 N_{c}[12]+2 C_{F}[34]
$$

with the same momentum labeling. The two terms correspond to gluon radiation off the initial state gluons (colour factor $N_{c}$ ) and the final-state quarks (colour factor $C_{F}$ ). With colour-singlet exchange in the $s$-channel (Fig. 1(b)), there is no interference between the initial- and final-state emission, in contrast to the QCD background antenna pattern. It is this feature which will give rise to significant quantitative differences between the radiation patterns (see below).
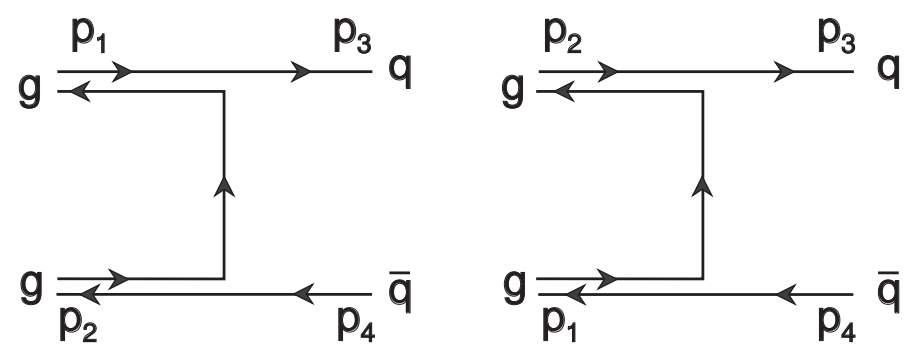

(a)

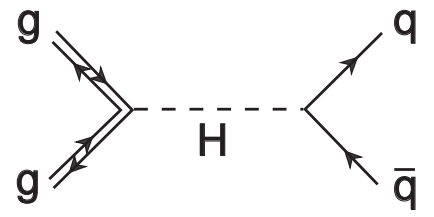

(b)

Figure 1: The colour flow diagrams for the processes (a) $g g \rightarrow q \bar{q}$ and (b) $g g \rightarrow H \rightarrow q \bar{q}$.

The next step is to define the kinematics. The four momenta are labelled by

$$
a\left(p_{1}\right)+b\left(p_{2}\right) \rightarrow c\left(p_{3}\right)+d\left(p_{4}\right)+g(k),
$$


where the gluon is assumed soft relative to the two large- $E_{T}$ partons $c$ and $d$, i.e. $k \ll$ $E_{T}$. Ignoring the gluon momentum in the energy-momentum constraints, working in the subprocess centre-of-mass frame, and using the notation $p^{\mu}=\left(E, p_{x}, p_{y}, p_{z}\right)$, we have

$$
\begin{aligned}
p_{1}^{\mu} & =\left(E_{T} \cosh \eta, 0,0, E_{T} \cosh \eta\right), \\
p_{2}^{\mu} & =\left(E_{T} \cosh \eta, 0,0,-E_{T} \cosh \eta\right), \\
p_{3}^{\mu} & =\left(E_{T} \cosh \eta, 0, E_{T}, E_{T} \sinh \eta\right), \\
p_{4}^{\mu} & =\left(E_{T} \cosh \eta, 0,-E_{T},-E_{T} \sinh \eta\right), \\
k^{\mu} & =\left(k_{T} \cosh (\eta+\Delta \eta), k_{T} \sin \Delta \phi, k_{T} \cos \Delta \phi, k_{T} \sinh (\eta+\Delta \eta)\right) .
\end{aligned}
$$

This is the appropriate form for studying the angular distribution of the soft gluon jet relative to the large $-E_{T}$ jet 3 , the separation between these being parametrised by $\Delta \eta$ and $\Delta \phi$. In terms of these variables, the soft gluon phase space is

$$
\frac{1}{(2 \pi)^{3}} \frac{d^{3} k}{2 E_{k}}=\frac{1}{16 \pi^{3}} k_{T} d k_{T} d \Delta \eta d \Delta \phi
$$

We will be particularly interested in the shape of the radiation pattern as a function of the variables $\Delta \eta$ and $\Delta \phi$. Note that the direction of the soft gluon is measured with respect to the $p_{3}$ jet. Thus for massless $2 \rightarrow 2$ scattering, collinear singularities are located at $\Delta \eta=0, \Delta \phi=0$ and $\Delta \eta=-2 \eta, \Delta \phi=\pi$.

We first study the QCD and Higgs radiation patterns for central $q \bar{q}$ jets, i.e. $\eta=0$. Using the kinematics of Eq. (8) with $\eta=0$, Eq. (6) gives

$$
\left.\mathcal{R}^{H}\right|_{\eta=0}=\frac{4}{k_{T}^{2}} \frac{N_{c}\left(\cosh ^{2}(\Delta \eta)-\cos ^{2}(\Delta \phi)\right)+C_{F}}{\cosh ^{2}(\Delta \eta)-\cos ^{2}(\Delta \phi)},
$$

and

$$
\begin{aligned}
\left.\mathcal{R}^{Q C D}\right|_{\eta=0} & =\frac{2}{k_{T}^{2}} \frac{N_{c}^{2}\left(2 \cosh ^{2}(\Delta \eta)-\cos ^{2}(\Delta \phi)-1\right)}{\left\{\left(4 C_{F}-N_{c}\right)\left(\cosh ^{2}(\Delta \eta)-\cos ^{2}(\Delta \phi)\right)\right\}} \\
& +\frac{4}{k_{T}^{2}} \frac{2 N_{c}\left(1-\cosh ^{2}(\Delta \eta)\right)+C_{F}\left(N_{c}^{2}-2\right)}{N_{c}\left\{\left(4 C_{F}-N_{c}\right)\left(\cosh ^{2}(\Delta \eta)-\cos ^{2}(\Delta \phi)\right)\right\}} .
\end{aligned}
$$

Note that the radiation patterns are independent of $E_{T}$. Fig. 2 shows the dependence of $\mathcal{R}^{H}$ and $\mathcal{R}^{Q C D}$ on $\Delta \eta$ and $\Delta \phi$. It is straightforward to show that the patterns are identical close to the beam direction,

$$
\lim _{|\Delta \eta| \rightarrow \infty} \mathcal{R}^{H, Q C D}=\frac{4}{k_{T}^{2}} N_{c}
$$



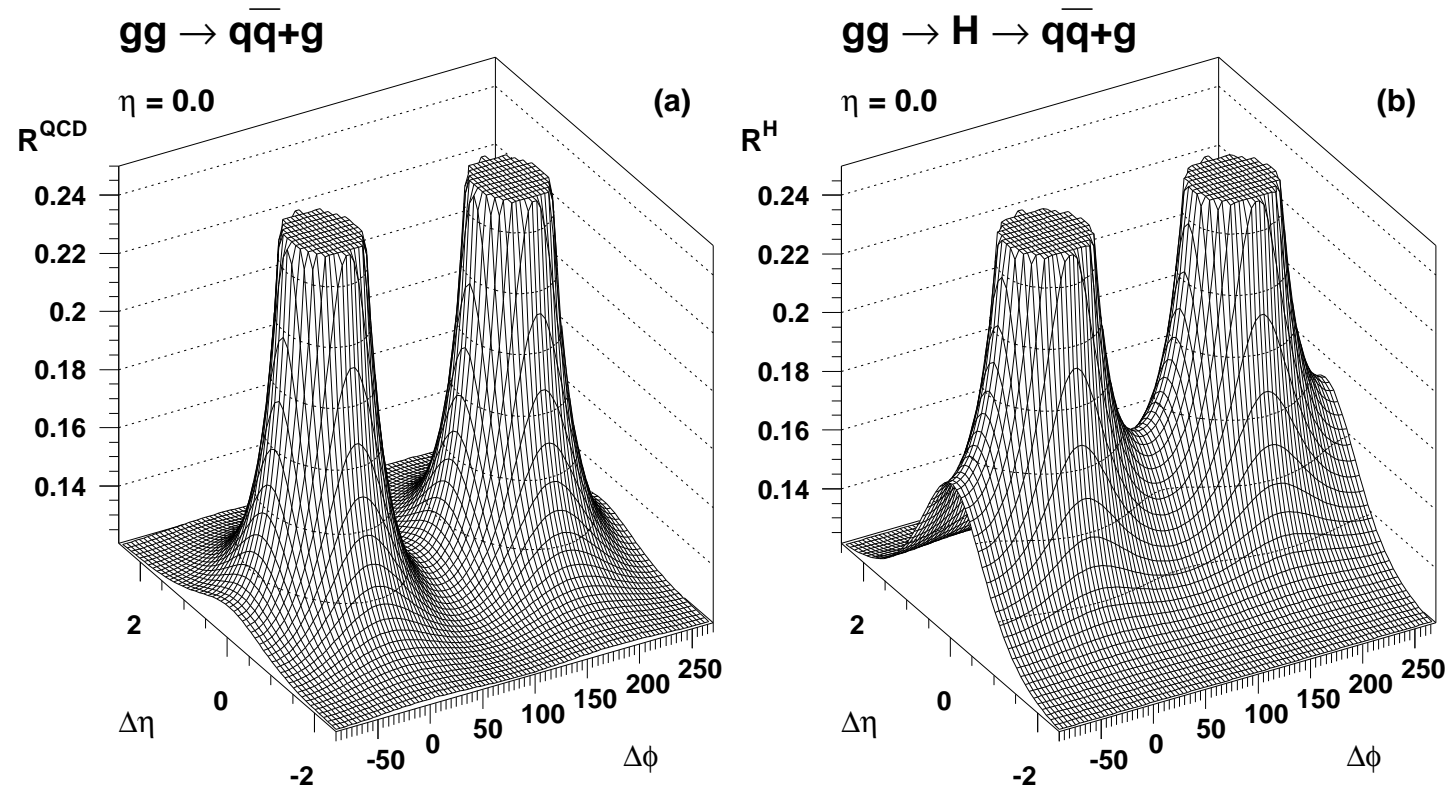

Figure 2: The antenna patterns $\mathcal{R}^{Q C D}$ of Eq. (5) and $\mathcal{R}^{H}=2 N_{c}[12]+2 C_{F}$ [34] of Eq. (6) for the processes $g g \rightarrow q \bar{q}+g$ and $g g \rightarrow H \rightarrow q \bar{q}+g$, with $\eta=0$ and $k_{T}=10 \mathrm{GeV}$. The units of $\mathcal{R}$ are $\mathrm{GeV}^{-2}$.

independent of $\Delta \phi$, and close to the directions of the final-state quarks,

$$
\lim _{\Delta \eta, \Delta \phi \rightarrow 0} \mathcal{R}^{H, Q C D} \rightarrow \frac{4 C_{F}}{k_{T}^{2}} \frac{1}{\cosh ^{2}(\Delta \eta)-\cos ^{2}(\Delta \phi)}
$$

The main difference arises from the amount of radiation between the final-state quark jets. To study this further we consider the distributions at the symmetric point $\mathcal{P}_{\mathrm{c}}$ located at $(\Delta \eta=-\eta=0, \Delta \phi=\pi / 2)$. This corresponds to soft gluon radiation perpendicular to the plane of the $g g \rightarrow q \bar{q}$ scattering, see Fig. 3. Again using the kinematics of Eq. (8), we find for the QCD background process

$$
\left.\mathcal{R}^{Q C D}\right|_{\eta=0}\left(\mathcal{P}_{\mathrm{c}}\right)=\frac{2}{k_{T}^{2}} \frac{2 C_{F}\left(N_{c}^{2}-2\right)+N_{c}^{3}}{N_{c}\left(4 C_{F}-N_{c}\right)} \sim 0.1304
$$

where the numerical value corresponds to $N_{c}=3$ and $k_{T}=10 \mathrm{GeV}$. In contrast, for the 
Higgs signal process we find

$$
\left.\mathcal{R}^{H}\right|_{\eta=0}\left(\mathcal{P}_{\mathrm{c}}\right)=\frac{4}{k_{T}^{2}}\left(C_{F}+N_{c}\right) \sim 0.1733
$$

There is therefore approximately $4 / 3$ more radiation between the final-state jets for the Higgs production process. This is due to the absence of a colour string connecting the final-state quarks in the QCD background process, see Fig. 1(a).

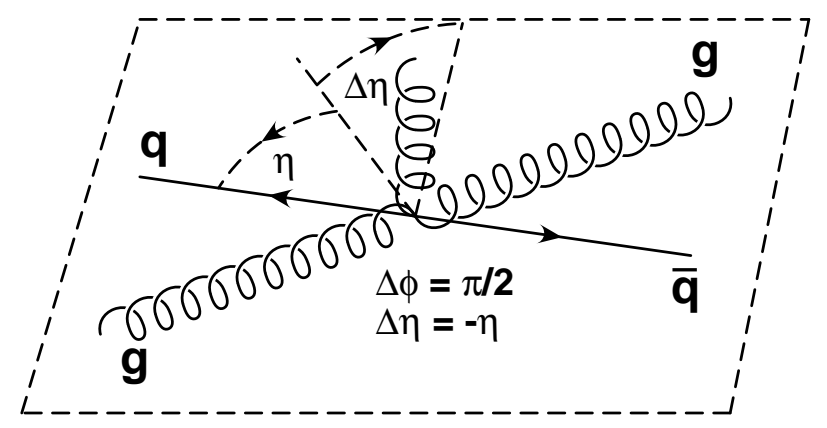

Figure 3: Sketch of the kinematics for the case of observing the soft gluon perpendicular to the event plane at the 'symmetric point' $\mathcal{P}_{\mathrm{c}}=(\Delta \eta=-\eta, \Delta \phi=\pi / 2)$.

The QCD background process does, however, have colour strings connecting the initial- and final-state quarks, and this leads to an enhancement of soft radiation between the jets in the plane of the scattering. We can illustrate this by considering the radiation patterns around the direction of the final state quark. In particular we introduce (as in [9]) the variables $(\Delta R, \beta)$, where

$$
\Delta \eta=\Delta R \cos \beta, \quad \Delta \phi=\Delta R \sin \beta .
$$

For fixed $\Delta R>0$, varying $\beta$ between 0 and $2 \pi$ describes a circle in the $(\Delta \eta, \Delta \phi)$ plane around the quark direction. In addition, if we fix $\Delta R=\pi / 2$ then the symmetric point $\mathcal{P}_{\mathrm{c}}$ corresponds to $\beta=\pi / 2$ (or equivalently $3 \pi / 2$ ), and the soft gluon is in the $2 \rightarrow 2$ scattering plane for $\beta=0, \pi$. Figure 4 shows the dependence of the radiation patterns $\mathcal{R}^{H, Q C D}$ on $\beta$ for $\Delta R=\pi / 2$, as before for $\eta=0$ final-state quarks. At $\beta=\pi / 2$ we have $\mathcal{R}^{H}>\mathcal{R}^{Q C D}$, as discussed above, whereas at $\beta=0, \pi$ we have $\mathcal{R}^{Q C D}=\mathcal{R}^{H}$. . The shape of the $\beta$ distribution therefore provides a powerful discriminator between signal and background.

\footnotetext{
${ }^{2}$ In fact, the equality of the distributions at $\beta=0, \pi$ is true for all $\Delta R$.
} 


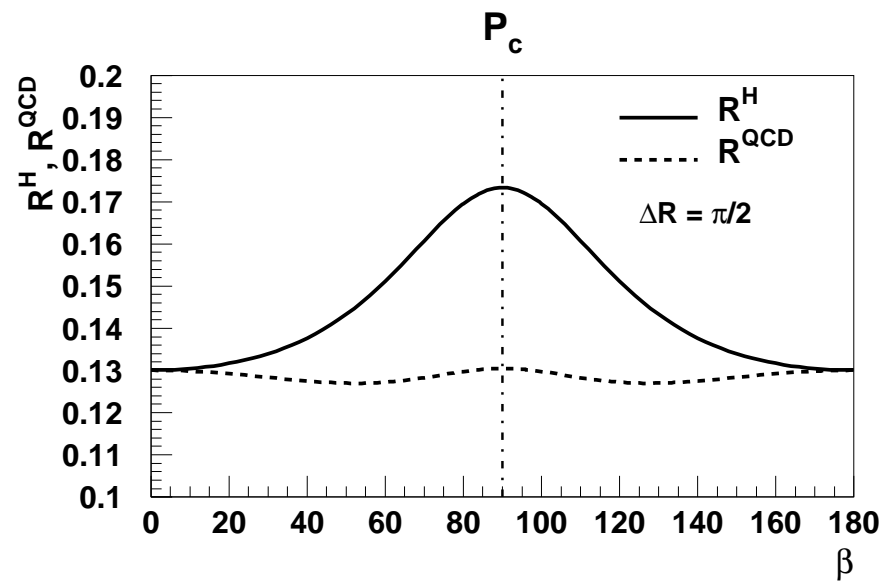

Figure 4: The dependence of the antenna patterns $\mathcal{R}^{H}$ and $\mathcal{R}^{Q C D}$ on the angular variable $\beta$ defined in Eq. (16). The units of $\mathcal{R}$ are $\mathrm{GeV}^{-2}$.

How does the interjet radiation enhancement depend on the jet rapidity $\eta$ ? Again we consider the symmetric point located at $\mathcal{P}_{\mathrm{c}}=(\Delta \eta=-\eta, \Delta \phi=\pi / 2)$. At this point $\mathcal{R}^{H}$ is completely independent of $\eta$,

$$
\mathcal{R}^{H}\left(\mathcal{P}_{\mathrm{c}}\right)=\frac{4}{k_{T}^{2}}\left(N_{c}+C_{F}\right) .
$$

which follows immediately from Eq. (6) since [12] $=[34]=2 / k_{T}^{2}$ at $\mathcal{P}_{\mathrm{c}}$. The result is slightly more complicated for $\mathcal{R}^{Q C D}$. Here we find

$$
\mathcal{R}^{Q C D}\left(\mathcal{P}_{\mathrm{c}}\right)=\frac{2}{k_{T}^{2}} \frac{1}{N_{c}} \frac{4 C_{F} \cosh ^{2}(\eta)\left(N_{c}^{2}-1\right)+N_{c}^{2}\left(N_{c}-2 C_{F}\right)}{4 C_{F} \cosh ^{2}(\eta)-N_{c}} .
$$

At $\mathcal{P}_{\mathrm{c}}, \mathcal{R}^{Q C D}$ is maximal for $\eta=0$ with the value given in Eq. (14). As $|\eta| \rightarrow \infty \mathcal{R}^{Q C D}$ approaches its minimum value,

$$
\lim _{|\eta| \rightarrow \infty} \mathcal{R}^{Q C D}\left(\mathcal{P}_{\mathrm{c}}\right)=\frac{4}{k_{T}^{2}} C_{F} .
$$

Note that in the large $\eta$ limit the ratio $\overline{\mathcal{R}} \equiv \mathcal{R}^{H} / \mathcal{R}^{Q C D}$ at $\mathcal{P}_{\mathrm{c}}$ is significantly larger than its value at $\eta=0$ :

$$
\overline{\mathcal{R}}\left(\eta=0, \mathcal{P}_{\mathrm{c}}\right)=\frac{3 N_{c}^{4}-7 N_{c}^{2}+2}{2 N_{c}^{4}-3 N_{c}^{2}+2}=1.3285,
$$




$$
\overline{\mathcal{R}}\left(|\eta| \rightarrow \infty, \mathcal{P}_{\mathrm{c}}\right)=\frac{3 N_{c}^{2}-1}{N_{c}^{2}-1}=3.25 .
$$

In other words, the difference in the signal and background radiation patterns at the symmetric interjet point increases with increasing jet rapidities. Note that the large $-N_{c}$ limits of the ratios in Eq. (20) are simply $3 / 2$ and 3 , and also that $\overline{\mathcal{R}}\left(\eta=0, \mathcal{P}_{\mathrm{c}}\right)=1$ for $N_{c}=2$. This is illustrated in Fig. 5 which shows the dependence of $\overline{\mathcal{R}}$ evaluated at $\mathcal{P}_{\mathrm{c}}$ on $\eta$ and $N_{c}$.

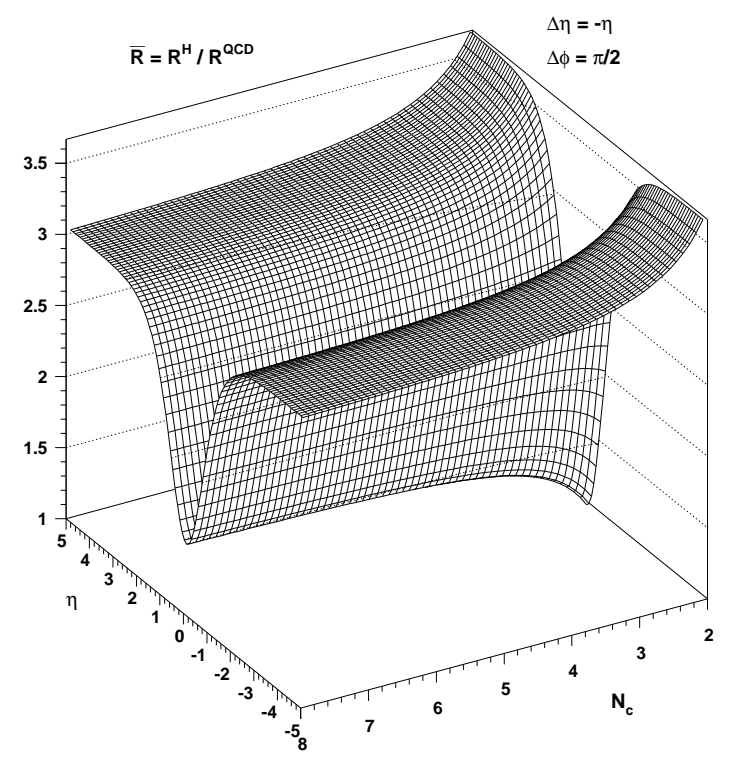

Figure 5: The ratio $\overline{\mathcal{R}}=\mathcal{R}^{H} / \mathcal{R}^{Q C D}$ as a function of $N_{c}$ and $\eta$ at the symmetric interjet point $\Delta \eta=-\eta$ and $\Delta \phi=\pi / 2$.

\subsection{Massive quarks}

So far we have only considered massless quarks. In fact for $H \rightarrow b \bar{b}$, with $m_{b} \ll M_{H}$, this should be an excellent approximation, since the soft gluon only 'feels' the finite $b$ quark mass very close to the jet axis, where our analysis does not in any case apply. Far from the jet direction, and in particular at the symmetric point $\mathcal{P}_{\mathrm{c}}$, the effect of the non-zero $b$ mass will be negligible. The situation is however very different for the case of $H \rightarrow t \bar{t}$, at $M_{H} \gtrsim 2 m_{t}$. Now mass effects are important in the radiation pattern, as we shall demonstrate below. 
If we allow a finite mass for the produced quarks then the kinematics have to be changed accordingly. Thus we replace the kinematics of Eq. (8) by

$$
\begin{aligned}
p_{1}^{\mu} & =\left(E_{q}, 0,0, E_{q}\right) \\
p_{2}^{\mu} & =\left(E_{q}, 0,0,-E_{q}\right) \\
p_{3}^{\mu} & =\left(E_{q}, 0, p_{T}, E_{q} \tanh \eta\right) \\
p_{4}^{\mu} & =\left(E_{q}, 0,-p_{T},-E_{q} \tanh \eta\right) \\
k^{\mu} & =\left(k_{T} \cosh (\eta+\Delta \eta), k_{T} \sin \Delta \phi, k_{T} \cos \Delta \phi, k_{T} \sinh (\eta+\Delta \eta)\right)
\end{aligned}
$$

i.e. we denote the energy of the quark jets by $E_{q}$ and their transverse momentum by $p_{T}$. Thus

$$
E_{q}=\cosh (\eta) \sqrt{m_{q}^{2}+p_{T}^{2}} .
$$

We again work in the subprocess centre-of-mass frame. It is convenient to introduce the dimensionless variable $\Theta$ as the ratio of the final-state quark mass $m_{q}$ to its energy

$$
\Theta=\frac{m_{q}}{E_{q}}
$$

For non-zero $m_{q}$ the antenna patterns receive additional contributions. For example, the antenna pattern of $\mathcal{R}^{H}$ of Eq. (6) becomes

$$
\mathcal{R}_{\Theta}^{H}=\mathcal{R}^{H}-C_{F}[33]-C_{F}[44],
$$

where the massive equivalents of $\mathcal{R}^{H}$ and $\mathcal{R}^{Q C D}$ are labelled with the suffix $\Theta$. One effect of the additional terms is to cancel the final-state collinear singularities, leading instead to the well-known dead cone [22] phenomenon. Using the results of Ref. [21], we obtain a somewhat more complicated expression for the massive equivalent to $\mathcal{R}^{Q C D}$,

$$
\begin{aligned}
\mathcal{R}_{\Theta}^{Q C D} & =\left(2 N_{c}-2 C_{F}+2 \mathcal{Y}\right)[12]+\left(C_{F}-\mathcal{X}-\mathcal{Y}\right)\{[13]+[24]\} \\
& +\left(C_{F}+\mathcal{X}-\mathcal{Y}\right)\{[14]+[23]\}+2 \mathcal{Y}[34]-C_{F}[33]-C_{F}[44]
\end{aligned}
$$

with

$$
\begin{aligned}
\mathcal{X} & =\frac{N_{c}^{2}}{4 C_{F}}\left[(1+2 \mu)\left(\frac{1}{U}-\frac{1}{T}\right)-\mu^{2}\left(\frac{1}{U^{2}}-\frac{1}{T^{2}}\right)+2(U-T)\right] \\
& \times\left[\frac{1}{U T}-\frac{N_{c}}{C_{F}}\right]^{-1}\left[T^{2}+U^{2}+2 \mu-\frac{\mu^{2}}{U T}\right]^{-1},
\end{aligned}
$$

and

$$
\mathcal{Y}=\frac{1}{4 C_{F}}\left[\frac{1}{N_{c}^{2} U T}+2\right]\left[\frac{1}{U T}-\frac{N_{c}}{C_{F}}\right]^{-1}
$$


The variables $T, U$ and $\mu$ are defined as

$$
T=\frac{p_{1} p_{3}}{p_{1} p_{2}}, \quad U=\frac{p_{1} p_{4}}{p_{1} p_{2}}, \quad \mu=\frac{m_{q}^{2}}{p_{1} p_{2}} .
$$

It is straightforward to show that the massless results are recovered in the limit $m_{q}(\Theta) \rightarrow$ 0 .

\subsection{Threshold behaviour $(\Theta=1)$}

We first study the behaviour of the radiation patterns $R_{\Theta}^{Q C D}$ and $R_{\Theta}^{H}$ in the threshold limit in which $m_{q}=E_{q}=M_{H} / 2$, i.e. $\Theta=1$. In fact setting $\eta=0$ we can readily derive the general expressions for the antennae for any value of $\Theta$. Figures 6 and 7 show the radiation patterns for various values of $\Theta$ near and at threshold. Notice how the strong peaking structure seen in the massless case (Fig. 22) disappears as the threshold is approached. In fact for $\Theta=1$, the patterns do not depend on $\Delta \phi$ at all. This can be seen from the analytic results. First, for $\Theta=1$ we have [34] $=[33]=[44]$ and so, from Eq. (6),

$$
\mathcal{R}_{\Theta=1}^{H}=2 N_{c}[12]=\frac{4}{k_{T}^{2}} N_{c}
$$

independent of $\Delta \eta$ and $\Delta \phi$, see Fig. T(d).

For $\mathcal{R}_{\Theta}^{Q C D}$ at threshold, we first note from (28) that $T=U=\mu=\frac{1}{2}$ and thus

$$
\mathcal{X}_{1}=0, \quad \mathcal{Y}_{1}=\frac{2+N_{c}^{2}}{2 N_{c}^{2}\left(4 C_{F}-N_{c}\right)} .
$$

From Eq. (25) we then have

$$
\begin{aligned}
\mathcal{R}_{\Theta=1}^{Q C D} & =2\left(N_{c}-C_{F}+\mathcal{Y}_{1}\right)[12]+\left(C_{F}-\mathcal{Y}_{1}\right)\{[13]+[24]+[14]+[23]\}+2\left(\mathcal{Y}_{1}-C_{F}\right)[34] \\
& =\frac{2}{k_{T}^{2}}\left(2 N_{c}-\frac{C_{F}-\mathcal{Y}_{1}}{\cosh ^{2}(\Delta \eta)}\right),
\end{aligned}
$$

which depends on $\Delta \eta$ but not on $\Delta \phi$. For $|\Delta \eta| \rightarrow \infty \mathcal{R}_{\Theta=1}^{Q C D}$ approaches the constant value

$$
\lim _{|\Delta \eta| \rightarrow \infty} \mathcal{R}_{\Theta=1}^{Q C D}=2 N_{c}[12]=\frac{4}{k_{T}^{2}} N_{c},
$$

and becomes equal to $\mathcal{R}_{\Theta=1}^{H}$, as in the massless case. We also see from Fig. 6(d) that $\mathcal{R}_{\Theta=1}^{Q C D}$ has an absolute minimum at $\Delta \eta=0$,

$$
\mathcal{R}_{\Theta=1}^{Q C D}(\Delta \eta=0)=\frac{2}{k_{T}^{2}}\left(2 N_{c}-C_{F}+\mathcal{Y}_{1}\right)=\frac{N_{c}}{k_{T}^{2}} \frac{3 N_{c}^{2}-4}{N_{c}^{2}-2},
$$




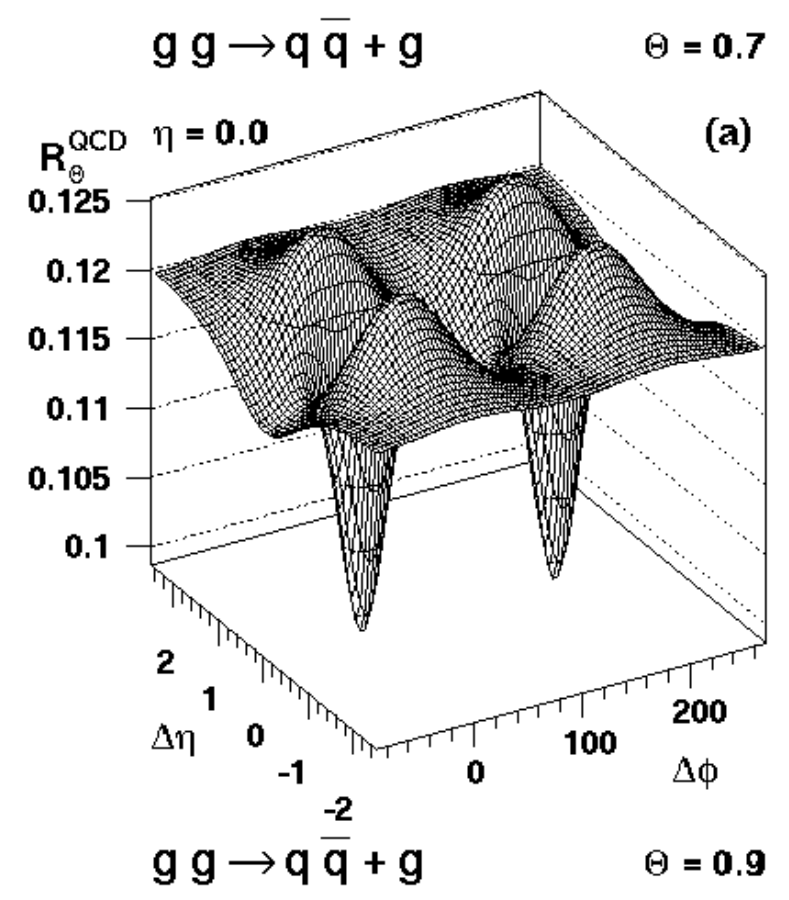

$g \mathrm{~g} \rightarrow \mathrm{q} \overline{\mathrm{q}}+\mathrm{g} \quad \Theta=0.8$
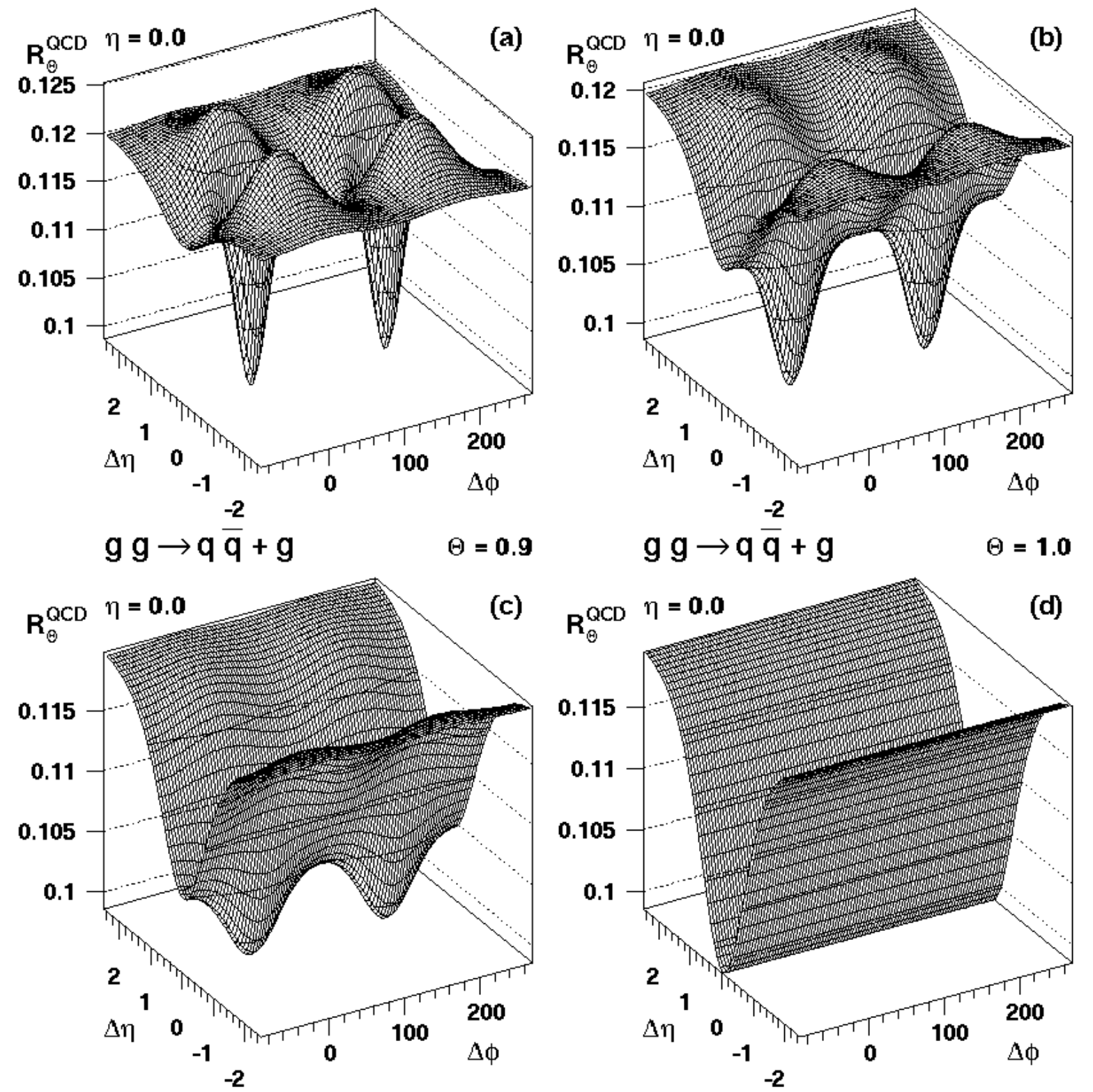

(b)
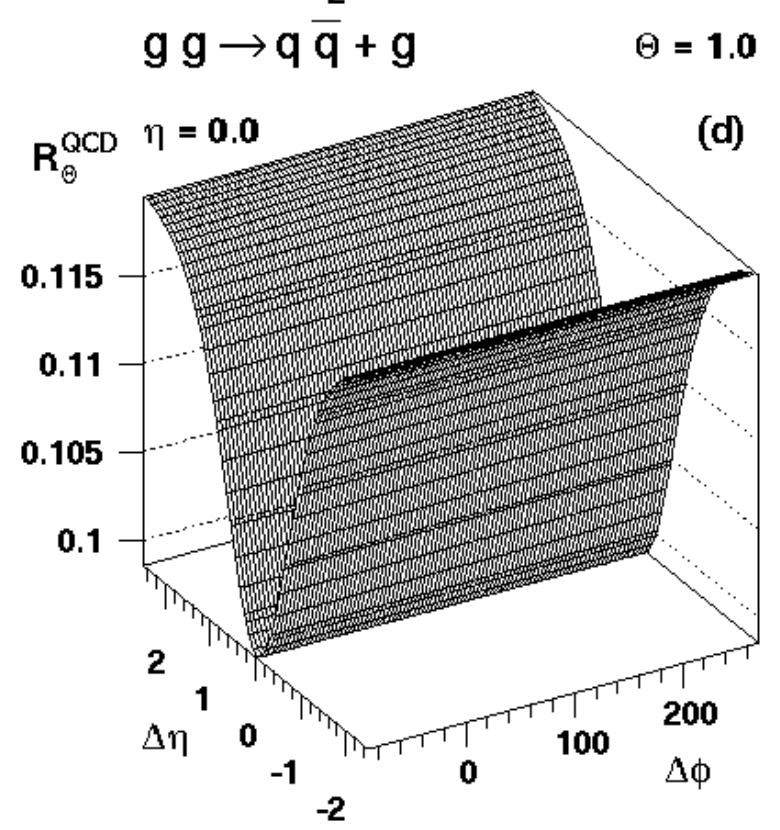

Figure 6: The antenna patterns $\mathcal{R}_{\Theta}^{Q C D}$ of Eq. (25) for the process $g g \rightarrow q \bar{q}+g$ with different values of the mass parameter $\Theta$ of Eq. (23). The pseudorapidity of both quark jets is fixed at $\eta=0$, and the transverse momentum of the soft gluon is $k_{T}=10 \mathrm{GeV}$. In (d) we show the threshold result $\Theta=1\left(E_{q}=m_{q}\right)$. The units of $\mathcal{R}_{\Theta}$ are $\mathrm{GeV}^{-2}$. 

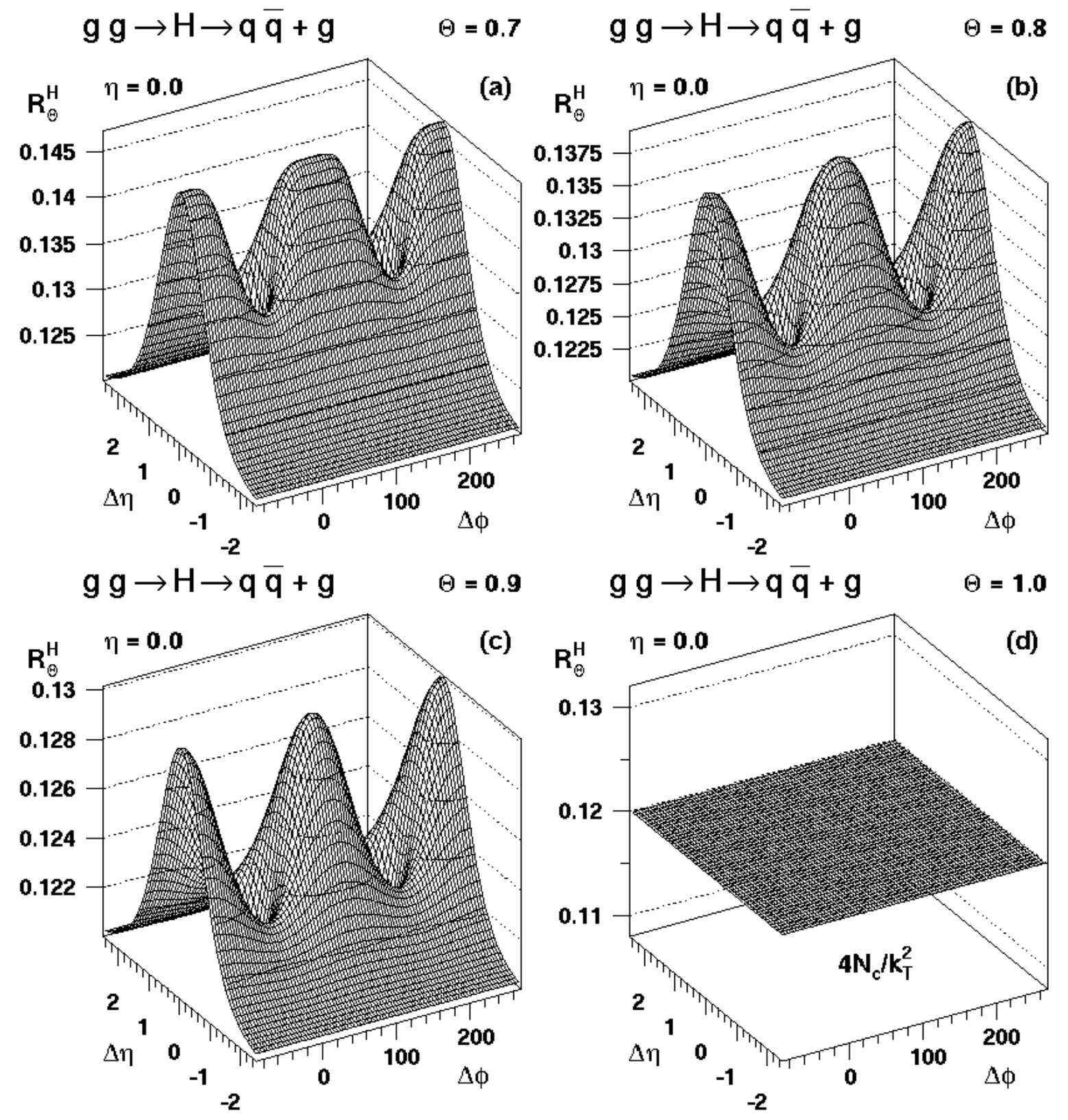

Figure 7: The antenna patterns $\mathcal{R}_{\Theta}^{H}$ of Eq. (25) for the process $g g \rightarrow H(\rightarrow q \bar{q})+g$ with different values of the mass parameter $\Theta$ of Eq. (23). The pseudorapidity of both quark jets is fixed at $\eta=0$, and the transverse momentum of the soft gluon is $k_{T}=10 \mathrm{GeV}$. In (d) we show the threshold result $\Theta=1\left(E_{q}=m_{q}\right)$. The units of $\mathcal{R}_{\Theta}$ are $\mathrm{GeV}^{-2}$. 
which is numerically $18 \%$ lower than the large $\Delta \eta$ value. Note the singularity in Eq. (33) for $N_{c}=\sqrt{2}$.

We next consider the patterns for arbitrary $\eta$ and $\Theta$. With the exception of [12] all antennae exhibit an $\eta$ dependence. We are again especially interested in the value of $\mathcal{R}_{\Theta}^{H}$ and $\mathcal{R}_{\Theta}^{Q C D}$ at the symmetric point between the two jets at $\mathcal{P}_{\mathrm{c}}=(\Delta \eta=-\eta, \Delta \phi=\pi / 2)$, as the massless study suggests that at this point the differences between the signal and background radiation patterns should be maximal. When evaluated at $\mathcal{P}_{\mathrm{c}}$, only [13], [14], [23] and [24] have an explicit $\eta$ dependence $(\sim \tanh (\eta))$, whereas

$$
\begin{aligned}
& {[12]=\frac{2}{k_{T}^{2}},} \\
& {[34]=\frac{2-\Theta^{2}}{k_{T}^{2}},} \\
& {[33]=[44]=\frac{\Theta^{2}}{k_{T}^{2}} .}
\end{aligned}
$$

All antennae that are $\eta$ dependent exhibit an absolute maximum at $\mathcal{P}_{\mathrm{c}}$ of $2 / k_{T}^{2}$ for $\eta \rightarrow$ $-\infty$ ([13], [24]) or for $\eta \rightarrow \infty([14]$, [23]) and vanish for $\eta \rightarrow \pm \infty$ accordingly. The fact that there is no $\eta$ dependence at $\mathcal{P}_{c}$ for [12], [34], [33] and [44] immediately yields (see Eq. (24))

$$
\mathcal{R}_{\Theta}^{H}\left(\mathcal{P}_{\mathrm{c}}\right)=\frac{4}{k_{T}^{2}}\left(N_{c}+C_{F}\left(1-\Theta^{2}\right)\right)
$$

for all $\eta$, i.e. the radiation between the two jets is completely independent of their separation in rapidity. This is illustrated in Fig. 8(a). Note that the massless result (Eq. (17)) is reproduced for $\Theta=0$. The corresponding expression for $\mathcal{R}_{\Theta}^{Q C D}$ is $\eta$ dependent and reads

$$
\mathcal{R}_{\Theta}^{Q C D}\left(\mathcal{P}_{\mathrm{c}}\right)=\frac{1}{k_{T}^{2}} \frac{\left\{4\left(1-\Theta^{2}\right)+2 N_{c}^{2}\left(N_{c}^{2}-2\right)\left(2-\Theta^{2}\right)\right\} \cosh ^{2}(\eta)+N_{c}^{2}\left\{2-\Theta^{2}\left(2-N_{c}^{2}\right)\right\}}{N_{c}^{2}\left(4 C_{F} \cosh ^{2}(\eta)-N_{c}\right)} .
$$

For fixed $\Theta, \mathcal{R}_{\Theta}^{Q C D}\left(\mathcal{P}_{\mathrm{c}}\right)$ always shows an absolute maximum for $\eta=0$ (see Fig. 8(b)) with a $\Theta$ dependence which again is maximal for the massless case $\Theta=0$, with the value given in Eq. (14). Once again defining the ratio of signal to background radiation patterns as $\overline{\mathcal{R}}_{\Theta}=\mathcal{R}_{\Theta}^{H} / \mathcal{R}_{\Theta}^{Q C D}$, we see that $\overline{\mathcal{R}}_{\Theta}$ has a local maximum at $\mathcal{P}_{\text {c }}$, the value of which depends on $\eta$ and $\Theta$, see Fig. $8(\mathrm{c})$. The value at $\eta=0$ is

$$
\overline{\mathcal{R}}_{\Theta}\left(\mathcal{P}_{c}, \eta=0\right)=\frac{4 N_{c}^{2}\left(4 C_{F}-N_{c}\right)\left(C_{F}\left(\Theta^{2}-1\right)-N_{c}\right)}{\left(N_{c}^{4}+4\right)\left(\Theta^{2}-1\right)+2 N_{c}^{2}\left(3-\Theta^{2}\right)},
$$

which actually shows a very weak $\Theta$ dependence. It is maximal for massless quarks $(\Theta=0)$ with the value $(=1.3285)$ already given in Eq. (20), and is minimal for $\Theta=1$ with the value

$$
\overline{\mathcal{R}}_{\Theta=1}\left(\mathcal{P}_{\mathrm{c}}, \eta=0\right)=4 \frac{N_{c}^{2}-2}{3 N_{c}^{2}-4}=1.2174 .
$$



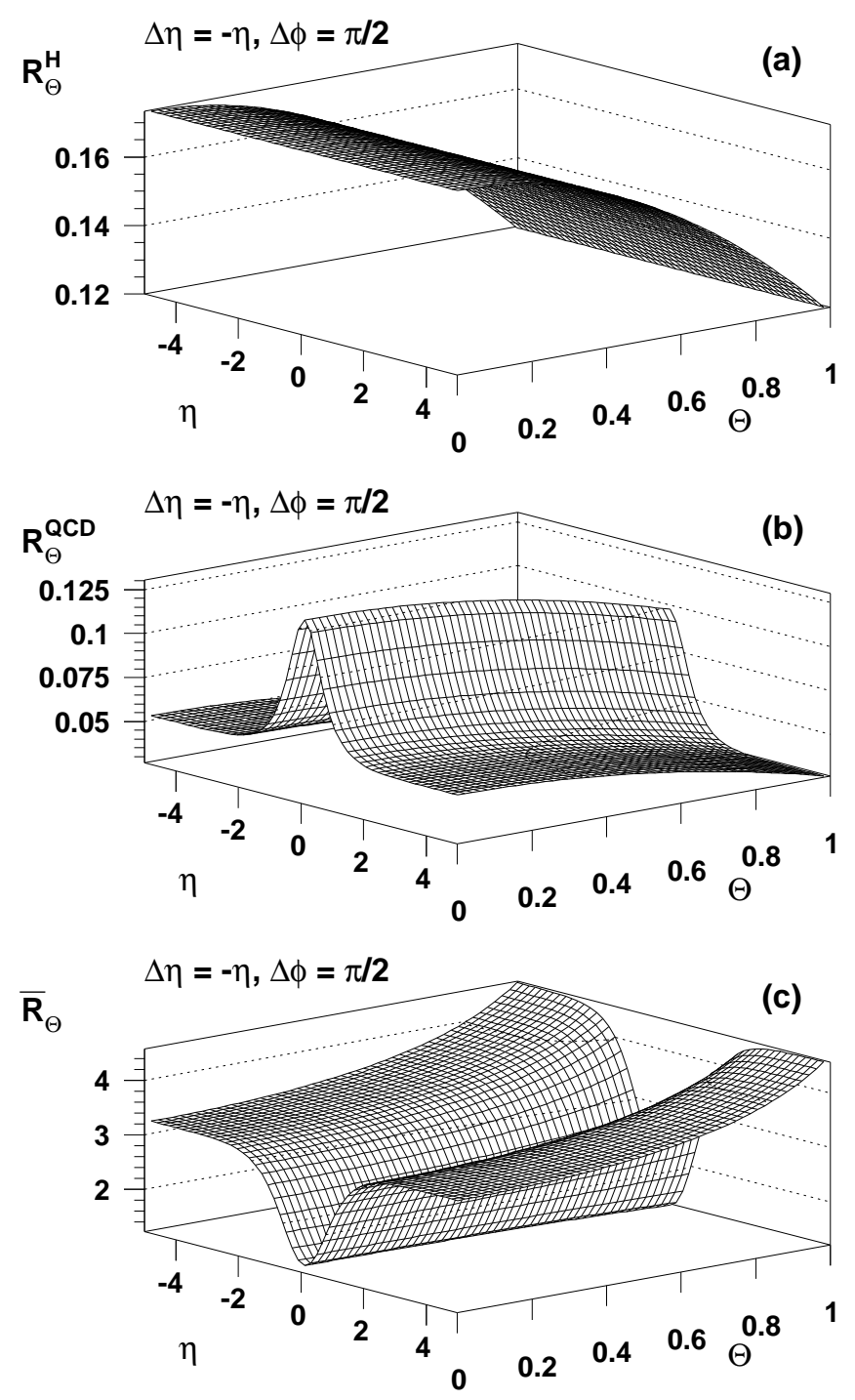

Figure 8: The antenna patterns for $\mathcal{R}_{\Theta}^{H}, \mathcal{R}_{\Theta}^{Q C D}$ (in units of $\mathrm{GeV}^{-2}$ ) and $\overline{\mathcal{R}}_{\Theta}$ at the symmetric interjet point $\mathcal{P}_{\mathrm{c}}$, for different values of the quark jet rapidity $\eta$ and the mass parameter $\Theta$. The soft gluon transverse momentum is taken to be $k_{T}=10 \mathrm{GeV}$. 
For the massless case, $\overline{\mathcal{R}}\left(\mathcal{P}_{\mathrm{c}}\right)$ increased with increasing jet separation (i.e. increasing $\eta)$. This is again true for the massive case, as shown in Fig. \&(c). In the limit $|\eta| \rightarrow \infty$ we find

$$
\lim _{|\eta| \rightarrow \infty} \overline{\mathcal{R}}_{\Theta}\left(\mathcal{P}_{c}, \eta\right)=\frac{4\left(N_{c}+C_{F}\left(1-\Theta^{2}\right)\right) N_{c}\left(N_{c}^{2}-1\right)}{2\left(1-\Theta^{2}\right)+N_{c}^{2}\left(N_{c}^{2}-2\right)\left(2-\Theta^{2}\right)}
$$

which is a monotonically increasing function of $\Theta$. The values at $\Theta=0,1$ are 3.25, 4.57 respectively, for $N_{c}=3$.

In summary, the relative difference between the radiation patterns for the Higgs signal and QCD background processes is maximal at the symmetric interjet point, as depicted in Fig. 3. The ratio (signal/background) of the radiation patterns at this point depends on the rapidity of the jets and the quark mass. It is smallest $(\overline{\mathcal{R}}=1.33)$ for massless, central jets, and largest for massive, large-rapidity jets $(\overline{\mathcal{R}}=4.57)$.

\subsection{Radiation inside the 'dead cone'}

A final point concerns the radiation inside the dead cone of the final-state (massive) quark jets. In this subsection for simplicity we will only consider centrally produced jets with $\eta=0$ - the generalisation to forward jet production is entirely straightforward.

First we recall the result for the Higgs signal process $g g \rightarrow H \rightarrow q \bar{q}$ for massless quarks (see Eq. (10)),

$$
\left.\mathcal{R}^{H}\right|_{\eta=0}=\frac{4}{k_{T}^{2}}\left(N_{c}+\frac{C_{F}}{\cosh ^{2}(\Delta \eta)-\cos ^{2}(\Delta \phi)}\right) .
$$

The second term is singular at the jet centre, $\Delta \eta, \Delta \phi \rightarrow 0$, whereas the first term represents a constant 'pedestal' of radiation from emission off the incoming gluons. In the massive case $(\Theta>0)$, however, the singularity is removed and in fact the net contribution to the radiation pattern from the combination $C_{F}(2[34]-[33]-[44])$ vanishes at the jet centre $\mathcal{P}_{\mathrm{dc}}=(\Delta \eta=\Delta \phi=0)$, hence

$$
\mathcal{R}_{\Theta}^{H}\left(\mathcal{P}_{\mathrm{dc}}, \eta=0\right)=\frac{4}{k_{T}^{2}} N_{c} .
$$

The corresponding result for the QCD background radiation pattern inside the dead cone is straightforward to calculate from the results already presented. We find, again for $\eta=0$,

$$
\mathcal{R}_{\Theta}^{Q C D}\left(\mathcal{P}_{\mathrm{dc}}, \eta=0\right)=\frac{N_{c}}{k_{T}^{2}} \frac{3 N_{c}^{2}-4}{N_{c}^{2}-2} .
$$

Interestingly, the results (41,42) are independent of the quark mass, provided of course that $m_{q}>0$. The effect can be seen in Figs. 6 and 7 , where the value of the radiation patterns at their minima (i.e. inside the dead cones of the quark jets) is the same for

all $\Theta$. The signal to background ratio in the dead cone is therefore equal to the value obtained at threshold and given already in Eq. (38). 


\section{Associated Higgs Production}

Higgs production in association with a W boson $q \bar{q}^{\prime} \rightarrow W^{*} \rightarrow W H$ is a potentially important discovery channel at both the Tevatron and LHC colliders, especially for the 'intermediate mass' Higgs. The non-hadronic final state $W H \rightarrow \ell \nu_{\ell} \gamma \gamma$ should be relatively easy to distinguish, but unfortunately has a very small branching ratio, see for example the recent study in Ref. [23]. This raises the question as to whether a search in the decay channel

$$
q \bar{q}^{\prime} \longrightarrow W^{*} \longrightarrow W\left(\rightarrow \ell \nu_{\ell}\right) H(\rightarrow b \bar{b})
$$

might be feasible, especially with flavour tagging of both final-state $b$ quarks [24]. Now there is a potentially large irreducible background from the QCD process

$$
q \bar{q}^{\prime} \longrightarrow W\left(\rightarrow \ell \nu_{\ell}\right)+b \bar{b}
$$

when $M_{b \bar{b}} \sim M_{H}$. The signal and background processes are illustrated in Fig. 9 .
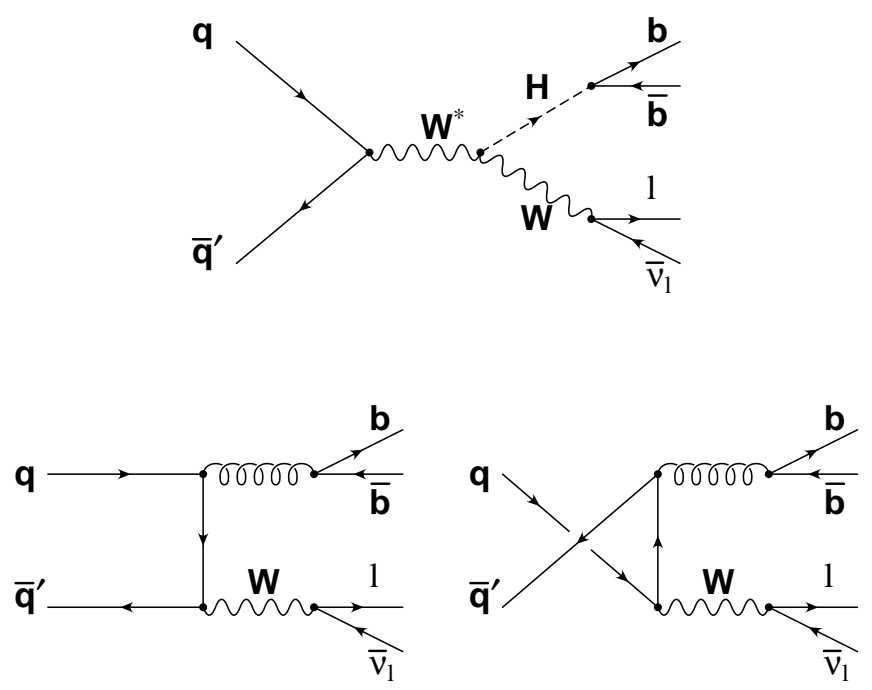

Figure 9: Feynman graphs for the process $q \bar{q}^{\prime} \rightarrow W^{*} \rightarrow W\left(\rightarrow \ell \bar{\nu}_{\ell}\right) H(\rightarrow q \bar{q})$ (associated Higgs production) and the background process $q \bar{q}^{\prime} \rightarrow W\left(\rightarrow \ell \bar{\nu}_{\ell}\right) g^{*}(\rightarrow q \bar{q})$.

We wish to study the radiation patterns for the processes (43) and (44), in analogy with the $g g \rightarrow(H \rightarrow) b \bar{b}$ study of the previous section. We first notice that the colour flows are exactly the same as those for the $2 \rightarrow 2$ scattering processes $q \bar{q} \rightarrow H \rightarrow b \bar{b}$ and $q \bar{q} \rightarrow g^{*} \rightarrow b \bar{b}$ [21]. We can therefore immediately write down the antenna patterns of the soft gluon radiation:

$$
\mathcal{R}_{\Theta}^{W H}=2 C_{F}\{[12]+[34]\}-C_{F}[33]-C_{F}[44] .
$$




$$
\mathcal{R}_{\Theta}^{W g}=\frac{1}{N_{c}}[14 ; 23]+2 C_{F}\{[13]+[24]\}-C_{F}[33]-C_{F}[44],
$$

with the momenta labelled as $q\left(p_{1}\right)+\bar{q}^{\prime}\left(p_{2}\right) \rightarrow W+b\left(p_{3}\right)+\bar{b}\left(p_{4}\right)$ and [14;23] defined in Eq. (3). Note that the Higgs pattern is the same as for $g g \rightarrow H \rightarrow b \bar{b}$ apart from colour factor replacement $N_{c} \rightarrow C_{F}$ for the initial-state [12] antenna.

In order to illustrate the quantitative differences between these radiation patterns it is necessary to define appropriate kinematics. Since the leading order processes are now effectively three-body final states, it is convenient to make some simplifying assumptions. Thus we assume that the $H$ and the $W$ are produced with zero rapidity, and that the $b$ and $\bar{b}$ quarks have equal energy and have polar and azimuthal angles $\vartheta_{b}$ and $\alpha_{b}$ with respect to the $H$ direction. This configuration is illustrated in Fig. 10 and corresponds to the four momenta

$$
\begin{aligned}
p_{1}^{\mu} & =(\sqrt{\hat{s}} / 2,0,0, \sqrt{\hat{s}}) \\
p_{2}^{\mu} & =(\sqrt{\hat{s}} / 2,0,0,-\sqrt{\hat{s}}) \\
p_{H}^{\mu} & =\left(E_{H}, p_{T H}, 0,0\right) \\
p_{W}^{\mu} & =\left(E_{W},-p_{T H}, 0,0\right) \\
p_{3}^{\mu} & =\left(E_{b}, p_{b} \cos \left(\vartheta_{b}\right), p_{b} \sin \left(\vartheta_{b}\right) \sin \left(\alpha_{b}\right), p_{b} \sin \left(\vartheta_{b}\right) \cos \left(\alpha_{b}\right)\right) \\
p_{4}^{\mu} & =\left(E_{b}, p_{T H}-p_{b} \cos \left(\vartheta_{b}\right),-p_{b} \sin \left(\vartheta_{b}\right) \sin \left(\alpha_{b}\right),-p_{b} \sin \left(\vartheta_{b}\right) \cos \left(\alpha_{b}\right)\right) .
\end{aligned}
$$

Conservation of energy and momentum gives

$$
E_{H}=2 E_{b}=\frac{\hat{s}+M_{H}^{2}-M_{W}^{2}}{2 \sqrt{\hat{s}}}, \quad p_{T H}=\sqrt{E_{H}^{2}-M_{H}^{2}}, \quad p_{b}=\sqrt{E_{b}^{2}-m_{b}^{2}}, \quad \cos \left(\vartheta_{b}\right)=\frac{p_{T H}}{2 p_{b}} .
$$

The pseudorapidities and azimuthal angles of the $b$ and $\bar{b}$ quarks are readily found to be

$$
\tan \left(\phi_{b, \bar{b}}\right)=\frac{p_{(b, \bar{b}) y}}{p_{(b, \bar{b}) x}}=\tan \left(\vartheta_{b, \bar{b}}\right) \sin \left(\alpha_{b, \bar{b}}\right)
$$

such that $\alpha_{b, \bar{b}}=\frac{\pi}{2}$ corresponds to $\phi_{b, \bar{b}}=\vartheta_{b, \bar{b}}$, and

$$
\eta_{b, \bar{b}}=\frac{1}{2} \ln \left(\frac{E_{b}+p_{(b, \bar{b}) z}}{E_{b}-p_{(b, \bar{b}) z}}\right) .
$$

The soft gluon momentum is defined relative to the $b$-quark jet:

$$
k^{\mu}=\left(k_{T} \cosh \left(\eta_{b}+\Delta \eta\right), k_{T} \cos \left(\phi_{b}+\Delta \phi\right), k_{T} \sin \left(\phi_{b}+\Delta \phi\right), k_{T} \sinh \left(\eta_{b}+\Delta \eta\right)\right) .
$$

Note that the opening angle $\left(2 \vartheta_{b}\right)$ between the two $b$ quarks is a function of the partonic subprocess energy $\sqrt{\hat{s}}$. The dependence is illustrated in Fig. 11. Note that at threshold $\left(\sqrt{\hat{s}}=M_{W}+M_{H}\right) 2 \vartheta_{b}=180^{\circ}$. 


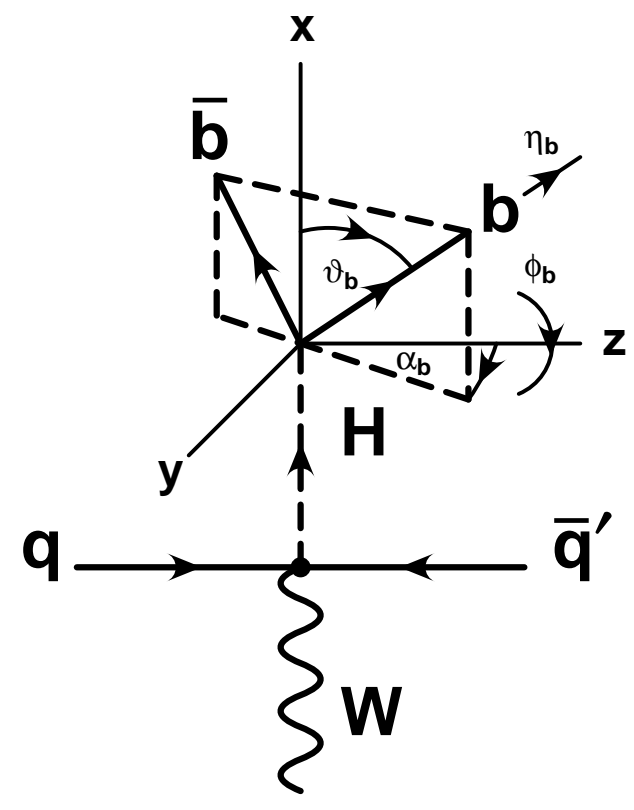

Figure 10: The kinematics for back-to-back Higgs $(\rightarrow b \bar{b})-W$ production. The variables are defined in Eq. (47).

Let us now study the radiation patterns in more detail. We assume parameter values of $M_{H}=130 \mathrm{GeV}, m_{b}=4.3 \mathrm{GeV}$ and $M_{W}=80.33 \mathrm{GeV}$, and we again fix the transverse momentum of the soft gluon to be $k_{T}=10 \mathrm{GeV}$. The first thing to note is that for the symmetric configuration defined above, the radiation pattern for the signal process is independent of the azimuthal angle $\alpha_{b}$. This follows from the absence of antenna involving both initial- and final-state quarks in (45). In contrast, there is no such symmetry for the background process (46).

A more striking difference is seen if we vary $\sqrt{\hat{s}}$. According to Fig. 11 the angle between the final-state quarks decreases with increasing $\sqrt{\hat{s}}$ with the effect that the two quark jets eventually merge for large centre-of-mass energies. Figs. 12 and 13 show the signal (45) and background (46) radiation patterns for the average value $(\sqrt{\hat{s}}=310 \mathrm{GeV})$ and for an extreme value $(\sqrt{\hat{s}}=14 \mathrm{TeV})$ respectively. The azimuthal angle $\alpha_{b}$ is fixed at $90^{\circ}$ in both cases. For $\sqrt{\hat{s}}=310 \mathrm{GeV}$ the opening angle between the $b$ and the $\bar{b}$ quarks is approximately $100^{\circ}$. As $\alpha_{b}=90^{\circ}$ the $b-\bar{b}$ plane is orthogonal to the $q \bar{q}^{\prime}-W H$

\footnotetext{
${ }^{3}$ Notice that at threshold, $\sqrt{\hat{s}}_{0}=M_{H}+M_{W}$, the $b$ and $\bar{b}$ are produced back-to-back, and the discussion is almost identical to the direct production case studied earlier, apart from colour factor differences arising from having incoming quarks instead of gluons.
} 


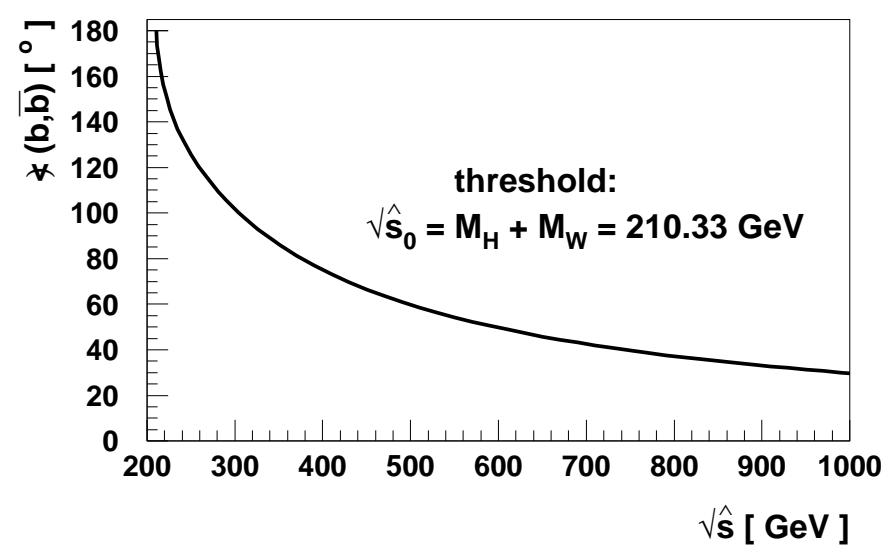

Figure 11: The opening angle of the $b \bar{b}$ quark pair as a function of the partonic subprocess energy $\sqrt{\hat{s}}$.

scattering plane (see Fig. 10) and thus $\eta_{b}=\eta_{\bar{b}}=0$. We see immediately that the main feature of our direct production study described earlier still holds. The most striking difference between the signal $\mathcal{R}_{\Theta}^{W H}$ and the background $\mathcal{R}_{\Theta}^{W g}$ is the relative suppression of radiation between the $b$-quark jets for the latter. There is a factor of approximately 2 difference between signal and background radiation in the interjet region, in qualitative agreement with the results obtained for direct Higgs production. If we now increase the subprocess centre-of-mass energy the two $b$-quark jets merge, forming a narrow colour singlet and octet state for the signal and background respectively. The situation for the extreme case $\sqrt{\hat{s}}=14 \mathrm{TeV}$ is shown in Fig. 13. Notice that for the signal process the soft gluon radiation becomes trapped in a very small tube. Outside the merged jets the radiation pattern completely flattens out. In contrast, for the background process there is still significant radiation between the initial- and final-state quark directions. In fact the distribution here is essentially identical to that for the $q \bar{q}^{\prime} \rightarrow W g$ process studied in Ref. [10]. In other words, the radiation pattern acts as a 'partonometer' [9] in measuring the colour charge of the outgoing large $p_{T}$ partonic system. 


\section{Conclusions}

We have studied the distribution of soft hadrons or jets accompanying the production and $q \bar{q}$ decay of light Higgs bosons at high-energy hadron colliders, and compared the distributions with those of the irreducible QCD backgrounds. We find significant differences between the signal and background distributions, which suggests that the study of the topology of hadron flow in such events could provide an important additional discriminatory tool. For example we have shown (see Fig. 2) that the distribution of soft hadrons transverse to the scattering plane in centrally produced $H \rightarrow b \bar{b}$ events is approximately $4 / 3$ larger than that for QCD $g g \rightarrow b \bar{b}$ events with the same kinematics, while close to the beam axis and final-state jet directions the signal and background distributions are the same. The differences result from the different colour flow in the two processes.

Although in this paper we have focused on light Higgs bosons with a dominant $b \bar{b}$ decay mode, we would like to make some additional remarks concerning heavier Higgs bosons. Consider, for example, the 'gold-plated' $g g \rightarrow H \rightarrow Z^{0} Z^{0} \rightarrow 4 l^{ \pm}$discovery channel for a heavy $\left(M_{H}>2 M_{Z}\right)$ Standard Model Higgs boson. The dominant irreducible background comes from the $q \bar{q} \rightarrow Z^{0} Z^{0}$ process. In the language of Section 2, the antenna patterns for these signal and background processes are simply $2 N_{c}[12]$ and $2 C_{F}[12]$ respectively. In other words, soft hadrons or jets with fixed transverse momentum should be uniformly distributed in the $(\eta, \phi)$ plane in both cases, but with an enhancement of $9 / 4$ for the signal relative to the background. A simple on-/off-resonance comparison should therefore show a significant difference.

Our results are based on the soft-gluon/LPHD hypothesis 13. The success of this approach has recently received a new quantitative confirmation from experiments at the Tevatron $p \bar{p}$ collider. However, it will be important to extend our work by incorporating a realistic Monte Carlo simulation which will allow detector effects to be included. We believe that the results presented in this paper make such an effort very worthwhile.

\section{Acknowledgements}

We thank John Ellis for useful discussions. This work was supported in part by the EU Fourth Framework Programme 'Training and Mobility of Researchers', Network 'Quantum Chromodynamics and the Deep Structure of Elementary Particles', contract FMRXCT98-0194 (DG 12 - MIHT).

\section{References}

[1] Yu.L. Dokshitzer, V.A. Khoze and S.I. Troyan, in Proc. 6th Int. Conf. on Physics in Collision, ed. M. Derrick (World Scientific, Singapore, 1987), p.417.

Yu.L. Dokshitzer, V.A. Khoze and S.I. Troyan, Sov. J. Nucl. Phys. 46 (1987) 712. 
[2] Yu.L. Dokshitzer, V.A. Khoze, A.H. Mueller and S.I. Troyan, Rev. Mod. Phys. 60 (1988) 373.

Yu.L. Dokshitzer, V.A. Khoze and S.I. Troyan in: Advanced Series on Directions in High Energy Physics, Perturbative Quantum Chromodynamics, ed. A.H. Mueller (World Scientific, Singapore), v. 5 (1989) 241.

[3] Yu.L. Dokshitzer, V.A. Khoze, A.H. Mueller and S.I. Troyan, "Basics of Perturbative QCD", ed. J. Tran Thanh Van, Editions Frontiéres, Gif-sur-Yvette, 1991.

[4] R.K. Ellis, G. Marchesini and B.R. Webber, Nucl. Phys. B286 (1987) 643; Erratum Nucl. Phys. B294 (1987) 1180.

R.K. Ellis, presented at "Les Rencontres de Physique de la Vallee d'Aoste", La Thuile, Italy, March 1987, preprint FERMILAB-Conf-87/108-T (1987).

[5] Yu.L. Dokshitzer, V.A. Khoze and S.I. Troyan, Sov. J. Nucl. Phys. 50 (1989) 505.

[6] Yu.L. Dokshitzer, V.A. Khoze and T. Sjöstrand, Phys. Lett. B274 (1992) 116.

[7] G. Marchesini and B.R. Webber, Nucl. Phys. B330 (1990) 261.

[8] D. Zeppenfeld, Madison preprint MADPH-95-933 (1996).

[9] J. Ellis, V.A. Khoze and W.J. Stirling, Zeit. Phys. C75 (1997) 287.

[10] V.A. Khoze and W.J. Stirling, Zeit. Phys. C76 (1997) 59.

[11] J. Amundson, J. Pumplin and C. Schmidt, Phys. Rev. D57 (1998) 527.

[12] V.A. Khoze, S. Lupia and W. Ochs, preprint CERN-TH/97-199, hep-ph/9711392.

[13] Ya.I. Azimov, Yu.L. Dokshitzer, V.A. Khoze and S.I. Troyan, Z. Phys. C27 (1985) 65; C31 (1986) 213.

[14] CDF collaboration: F. Abe et al., Phys. Rev. D50 (1994) 5562.

[15] D0 collaboration: B. Abbott et al., Phys. Lett. B414 (1997) 419; preprint FERMILAB-Conf-97-372-E; N. Varelas, preprint FERMILAB-Conf-97-346-E.

[16] Proceedings of the "Large Hadron Collider Workshop", Aachen, 4-9 October 1990, eds. G. Jarlskog and D. Rein, Report CERN 90-10, ECFA 90-133, Geneva, 1990.

[17] ATLAS Technical Proposal, CERN/LHC/94-43 LHCC/P2 (December 1994).

[18] CMS Technical Proposal, CERN/LHC/94-43 LHCC/P1 (December 1994).

[19] M. Heyssler and W.J. Stirling, Phys. Lett. B407 (1997) 259. 
[20] Ya.I. Azimov, Yu.L. Dokshitzer, V.A. Khoze and S.I. Troyan, Phys. Lett. B165 (1985) 147.

[21] V.A. Khoze, J. Ohnemus and W.J. Stirling, Phys. Rev. D49 (1994) 1237.

[22] Yu.L. Dokshitzer, V.A. Khoze and S.I. Troyan, J. Phys. G17 (1991) 1481; ibid. G17 (1991) 1602; Phys. Rev. D53 (1996) 89.

[23] Z. Kunszt, S. Moretti and W.J. Stirling, Zeit. Phys. C74 (1997) 479.

[24] A. Stange, W. Marciano and S. Willenbrock, Phys. Rev. D50 (1994) 4491. 


$$
\begin{array}{llll} 
& \multicolumn{1}{c}{\hat{s}=310 \mathrm{GeV}, \alpha_{b}=90^{\circ}} & \sqrt{s}=310 \mathrm{GeV}, \alpha_{b}=90^{\circ} \\
\eta_{b}=\mathbf{0 . 0} & \eta_{b}=\mathbf{0 . 0} & \\
\phi_{b}=49.029^{\circ} & \text { (a) } & \phi_{b}=49.029^{\circ} & \text { (b) }
\end{array}
$$

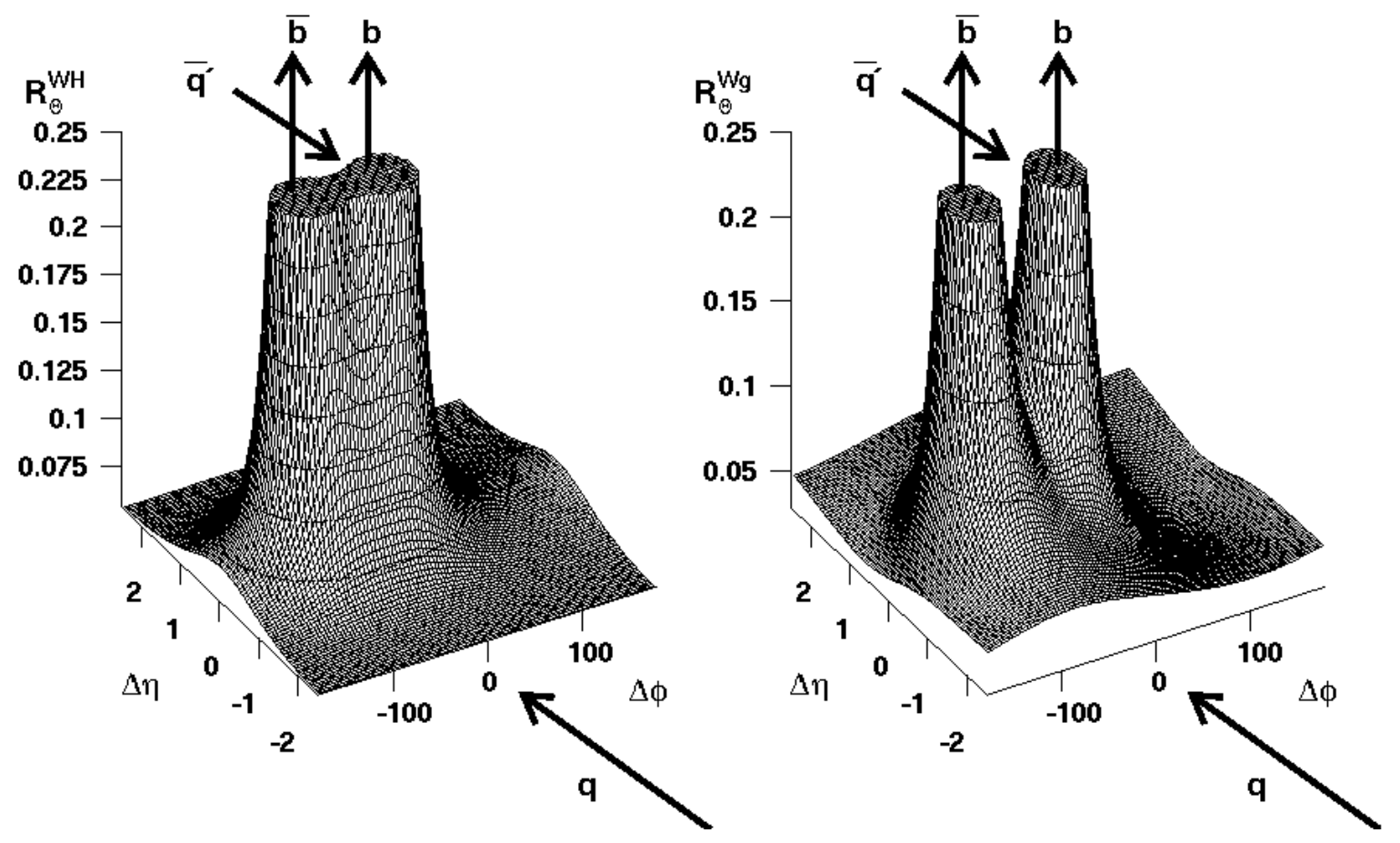

Figure 12: The antenna patterns (in units of $\mathrm{GeV}^{-2}$ ) for the signal $\mathcal{R}_{\Theta}^{W H}$ (Eq. (45)) and the background $\mathcal{R}_{\Theta}^{W g}$ (Eq. (46)) for associated Higgs production at subprocess centre-ofmass energy $\sqrt{\hat{s}}=310 \mathrm{GeV}$. The directions of the incoming quarks $q$ and $\bar{q}^{\prime}$ and of the $b$ and $\bar{b}$ quarks are indicated. 


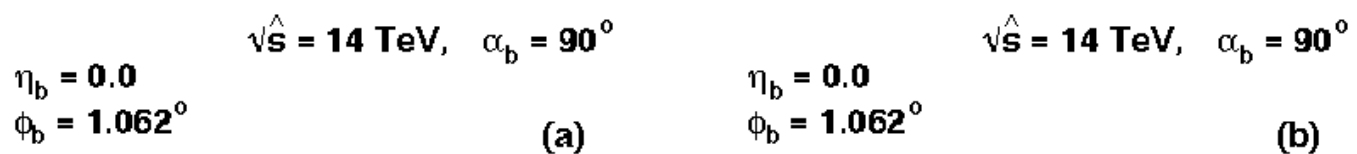

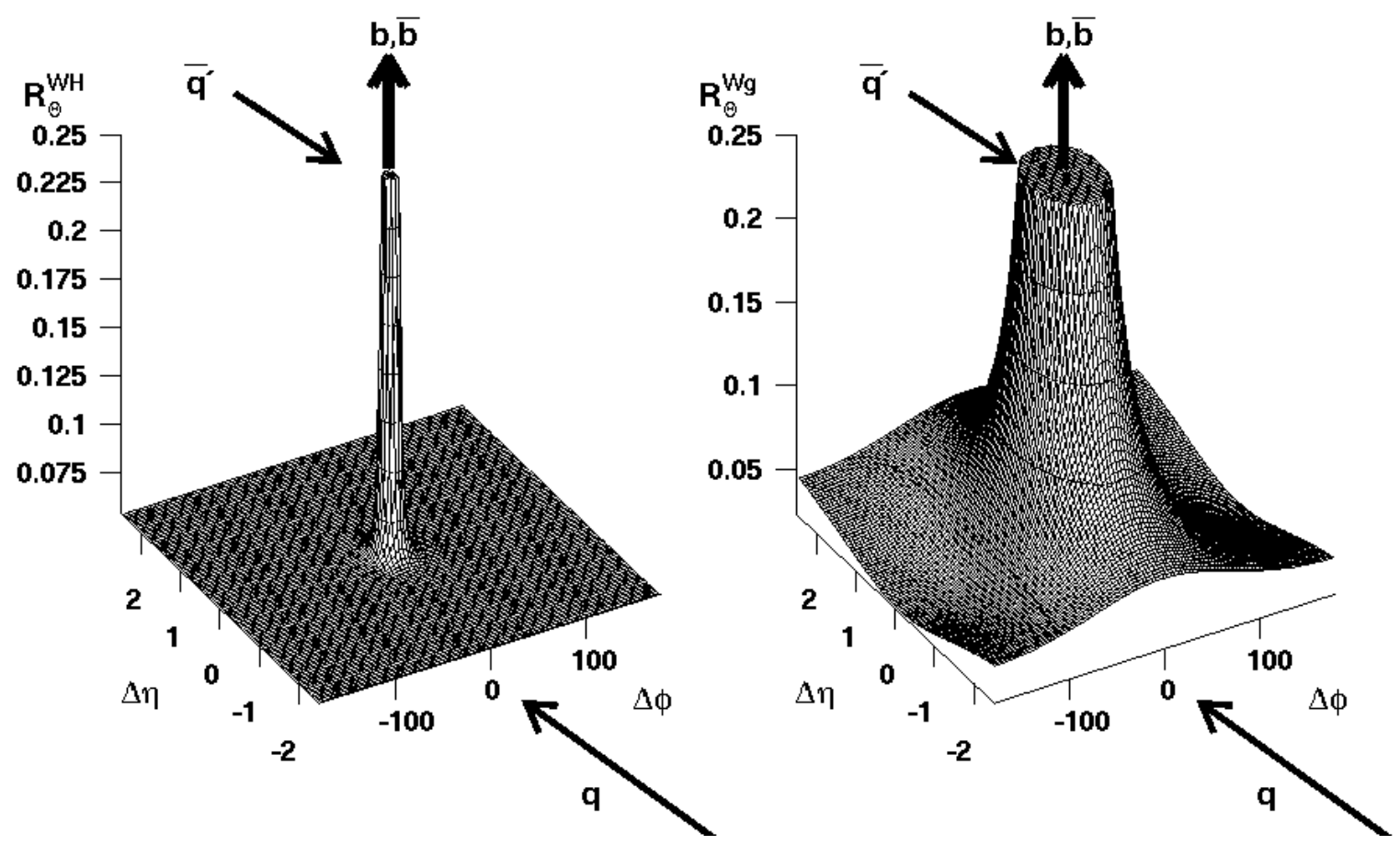

Figure 13: Same as Fig. 12 but now for a subprocess centre-of-mass energy of $\sqrt{\hat{s}}=$ $14 \mathrm{TeV}$. 